\title{
Study of Multi-Level Cryptography Algorithm: Multi-Prime RSA and DES
}

\author{
Surinder Kaur \\ Bharati Vidyapeeth's College of Engineering, Department of Information Technology, Delhi, 110063, India \\ E-mail: kaur.surinder@bharatividyapeeth.edu \\ Pooja Bharadwaj and Shivani Mankotia \\ Bharati Vidyapeeth's College of Engineering, Department of Information Technology, Delhi, 110063, India \\ E-mail: bharadwajp@acm.org, mankotias@acm.org
}

Received: 28 April 2017; Accepted: 05 July 2017; Published: 08 September 2017

\begin{abstract}
The purpose of this study is to implement and observe parameters like time and memory for implementation of multi-level encryption using the Data Encryption Standard (DES) and a modified version of the RSA Algorithm, the multi-prime RSA. The average values are calculated for each parameter after using a different number of primes and the results have been illustrated graphically and in tabular form for clarity of conclusions. The advantages and reasoning for using this approach have also been listed in the study.
\end{abstract}

Index Terms-RSA algorithm, DES, encryption, decryption, n-prime RSA, Data Encryption Standard, Multi-level encryption.

\section{INTRODUCTION}

The primary objective of this study is to understand and implement multi-level encryption using RSA and DES. A sample plaintext data is encrypted using a combined approach of the two algorithms. Decryption is performed accordingly and the time and memory parameters are noted. The RSA algorithm has been one of the most popular asymmetric algorithms for public key cryptography.

The strength of the RSA algorithm has been the difficulty of extracting two large prime numbers from their product, i.e., factorization of the large number obtained from their multiplication [1].

This strength was further utilized by implementation of the multi prime RSA algorithm, wherein, more than 2 prime numbers are used to generate the private and public key [2]. This implementation leads to a substantial increase in decryption time with increase in the number of primes.

Since RSA is an old algorithm, using it to transfer large amounts of sensitive or confidential data is not pragmatic. Hence, the DES algorithm is used to encrypt the raw data. Another layer of encryption is added on the DES key using the RSA algorithm [4]. This solves the problem of DES symmetric key secure sharing. This can be shown this form of a block diagram in figure 1 [5].
"DES: (Data Encryption Standard), was the first encryption standard to be recommended by NIST (National Institute of Standards and Technology)." DES has a key size of 64 bits and block size, also 64 bits. Many attacks and analysis have explored the weaknesses of DES, which make it somehow a vulnerable form of block cipher [6] [7].

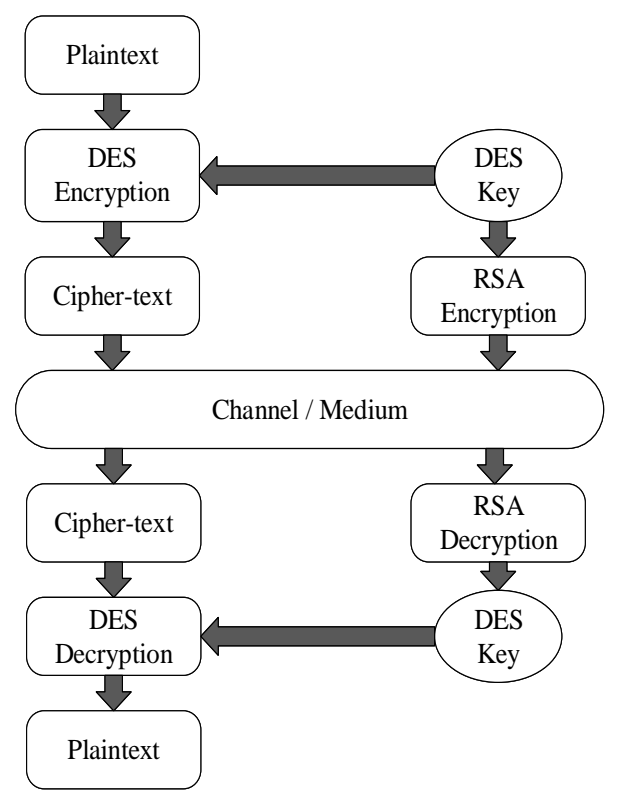

Fig.1. Multi-Level RSA and DES Block Diagram.

Triple DES is a stronger variant of DES. It also has a 64-bit block size along with 192 bits of the key size. The encryption process in Triple DES is same as that of DES, but the whole set of steps is applied three times for more security. A combination of different keys are used for implementing Triple DES [6].

In this paper, we will implement a combination of the standard RSA and DES algorithm, and then substitute RSA with its variant, the multi-prime RSA. The rest of the paper includes the following; a brief Literature Review of this algorithm and its variations is given in section II. Section III is about the standard working of RSA Algorithm with DES. Section IV discusses the 
methodology used in implementing n-prime RSA with DES with comparisons of encryption and decryption times with standard RSA. It also includes memory consumption averages as another comparison parameter. Section V discusses results and graphical representations of the data collected. Sections VI and VII discuss the future scope and limitations of our research, respectively, and Section VIII concludes the paper.

\section{RELATED WORK AND IMPLEMENTATIONS}

The idea if combining RSA and DES to reap the benefits of both symmetric and asymmetric key encryption is not a new one. Security, being a prime issue has always been researched and experimented extensively. Below are some recent related work and implementations that use the Hybrid Cryptography approach.

A framework for data security was proposed in 2016, where separate frames were designed for client and server. A user registration process was included on the server side for authorization. The approach included 3 phases to be applied on the data, namely, DES, RSA, Hybrid algorithm. The keys that were used in the last step were string, modulus, private, public and integer key [8].

Another approach was proposed for combining symmetric and public key methods in 2012. Again, AES and DES were used for data encryption and RSA was used for key exchange. The system comprising of many modules was modelled using Verilog HDL using Mdel Sim SE 5.7e. It also consisted of a pseudorandom number generation unit for the key generation process in addition to a GCD computation unit to be used in the RSA algorithm [9].

Aiming ay user's integrity, authentication and accuracy, this approach uses two different cryptographic algorithms for both encryption and decryption. A public key cryptographic algorithm based on linear block cipher and other is based on symmetric cryptography algorithm [10].

Multilevel and hybrid cryptography algorithms are used to enhance the security of cloud database, for Database-as-a-service. The cryptography tools used are RSA, DES and Random Number Generator. This technique is more profound on small amount of data like passwords [11].

Hybrid algorithm based on RSA and DES is put forward to increase the Data Transmission security in Bluetooth communications. Instead of the current encryption method in Bluetooth, that is, E0 encryption, which is 128-bit symmetric stream cipher, DES and RSA are used. DES being a block encryption, provides higher data transmission efficiency and RSA encrypts the DES keys. This combination makes the Bluetooth Transmission more reliable and secure than E0 encryption [12].

\section{Multi-LEVEL CRYPTOGRAPHY}

Multi-level cryptography was implemented using DES and RSA, and subsequently using DES and Multi-prime RSA.

The steps followed for the same are illustrated in the following flow charts [4] [13]:

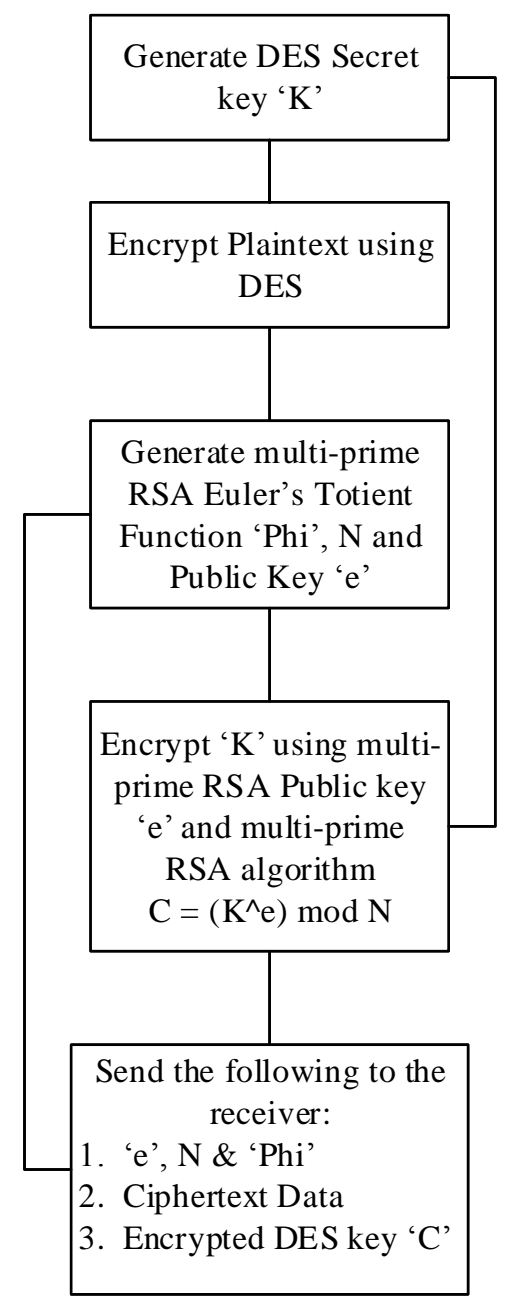

Fig.2. Multi-Level Encryption on Sender Side.

\section{A. Encryption}

At the sender side, encryption is done as follows [4] [13]:

- Generate DES Secret key. (Since DES is a symmetric key algorithm, only one key is generated and shared).

- Use this Secret Key to encrypt the plaintext data using DES.

- Generate RSA Public Key 'e'.

- Use this key to encrypt the DES Secret Key using the RSA algorithm.

- The following information is shared with the receiver.

1. RSA Public Key 'e' and (Euler's Totient function), product of primes $\mathrm{N}$.

2. Encrypted Data

3. Encrypted DES Secret key 


\section{B. Decryption}

At the receiver side, Decryption is done as follows:

- Generate the private key, ' $d$ ' using $\Phi$ and 'e'.

- Use this key to decrypt the DES secret key using RSA.

- The secret key thus obtained is then used to decrypt the cipher-text data using DES.

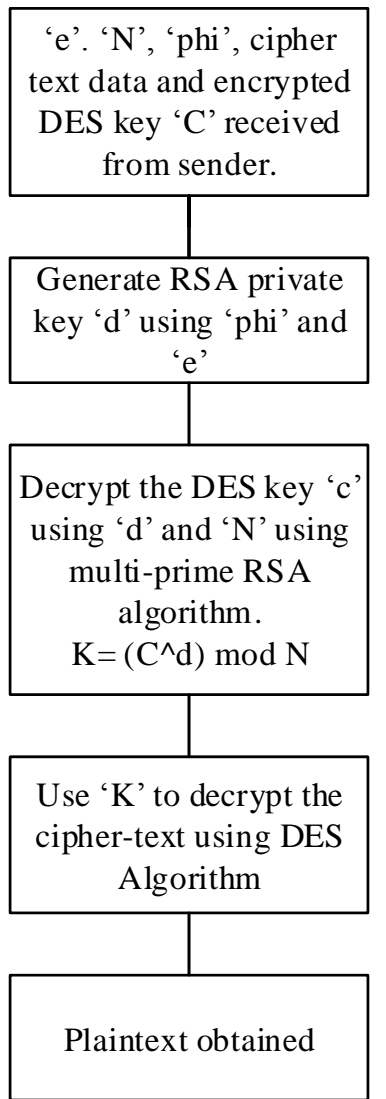

Fig.3. Multi-level Decryption on Receiver Side.

This implementation does two things, one, it encrypts the DES secret key using RSA, and two, it encrypts the main data to be transmitted using DES.

Hence, at the receiver side, decryption also needs to be done at two levels. The first step is to decrypt the RSA encrypted DES key, and the second is to decrypt the actual data using the key obtained in the first step.

It is important to note here that RSA in isolation is not a strong encryption strategy whereas DES is. Combining the two might not necessarily increase the strength of the security of the data, but definitely makes it more difficult and time consuming for any potential attacker. This technique combined with other detecting mechanisms can help in the identification of an attack before it actually escalates to a dangerous level.

Following results were obtained when DES and Standard RSA was implemented in JAVA:

Average running time for encryption: $14.133333 \mathrm{~ms}$ Average running time for decryption: $31.333333 \mathrm{~ms}$ Average Memory consumption: 24.08738556 MB

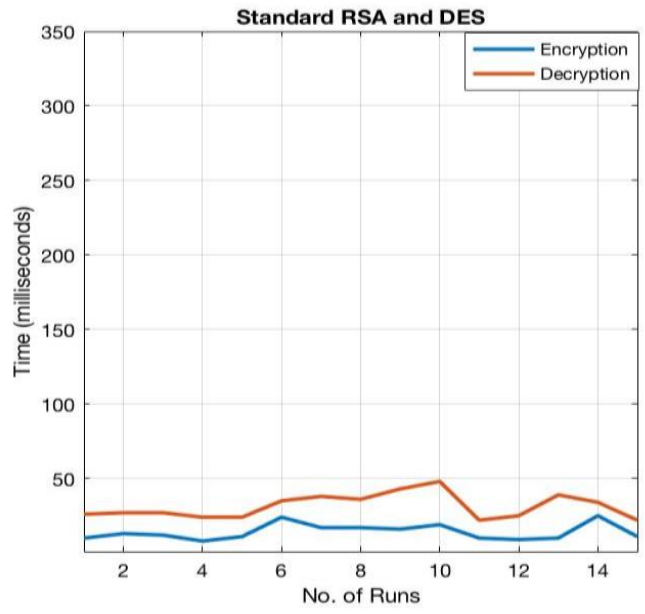

Fig.4. Encryption/Decryption Times for Standard RSA+DES

\section{METHODOLOGY}

The problem of key sharing in the DES arises due to the symmetric nature of the algorithm. By using an asymmetric algorithm to encrypt the shared DES key, this concern can be eliminated [14]. Further, the approach here is to see how practical and feasible it is to apply the multi prime version of RSA in combination with DES.

The following sections cover implementation and the average encryption and decryption times of 3-prime, 4prime and 5-prime RSA with DES, and the average memory consumption of DES+RSA with 2-10 prime numbers used.

We have used bit length of 1024 and 256 bytes is the block size, the time has been recorded in milliseconds (ms).

\section{A. DES and 3-Prime RSA}

The 3-prime RSA encryption is used in place of the standard RSA. The DES algorithm remains the same. Hence, the DES key is encrypted using 3-prime RSA.

Average running time for encryption: $18.1 \mathrm{~ms}$ Average running time for decryption: $77.8 \mathrm{~ms}$ Average memory consumption: 27.12103221 MB

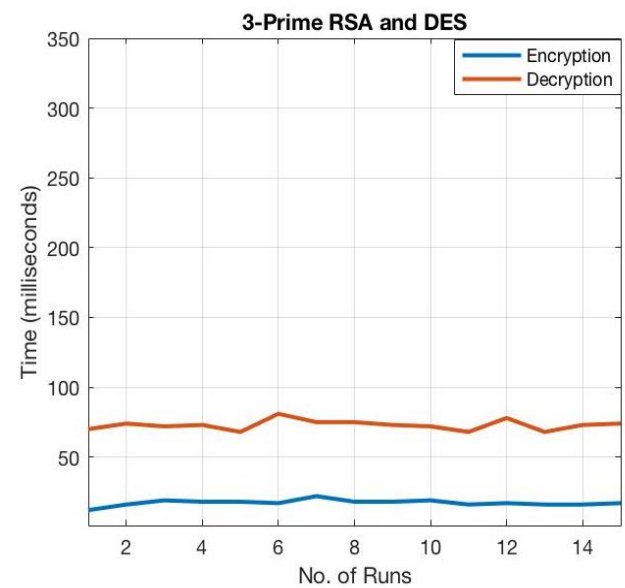

Fig.5. Encryption/Decryption Times for 3-prime RSA+DES. 


\section{B. DES and 4-Prime RSA}

The 4-prime RSA encryption is used in place of the standard RSA. DES encryption/decryption remains the same.

Average running time for encryption: $18.9 \mathrm{~ms}$ Average running time for decryption: $163.7 \mathrm{~ms}$

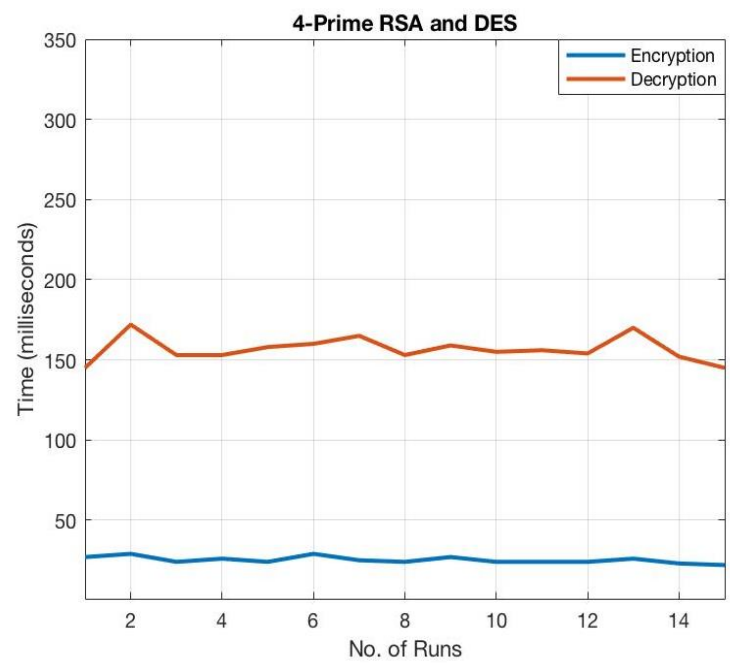

Fig.6. Encryption/Decryption Times for 4-prime RSA+DES.

\section{DES and 5-Prime RSA}

The 5-prime RSA encryption is used in place of the standard RSA. DES encryption/decryption remains the same.

Average running time for encryption: $24.7 \mathrm{~ms}$ Average running time for decryption: $312.9 \mathrm{~ms}$ Average memory consumption: $15.43968793 \mathrm{MB}$

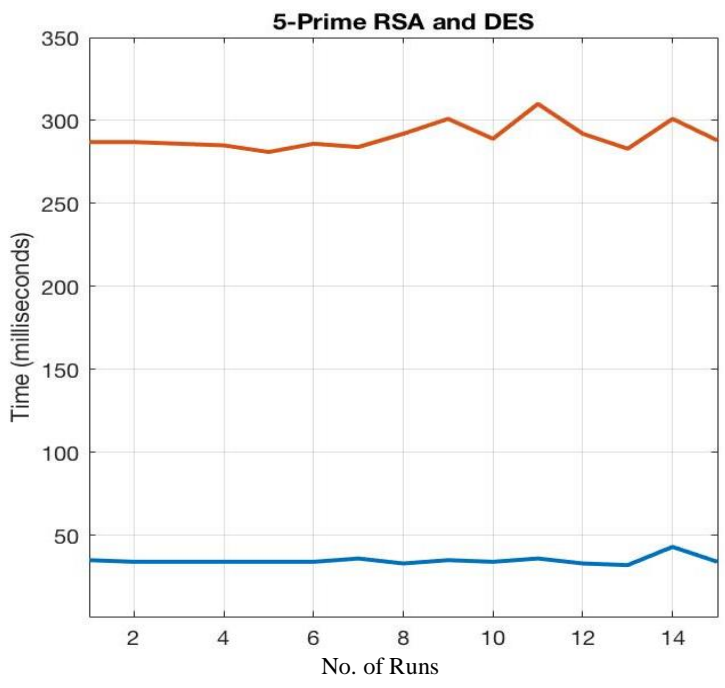

Fig.7. Encryption/Decryption Times for 5-prime RSA+DES.

After 5 prime numbers, to proceed further, we reset the Y- axis upper limit to a much higher value for the purpose of clarity in comparison.

\section{DES and 6-Prime RSA}

The 6-prime RSA encryption is used in place of the Standard RSA. The DES encryption/decryption remains the same.

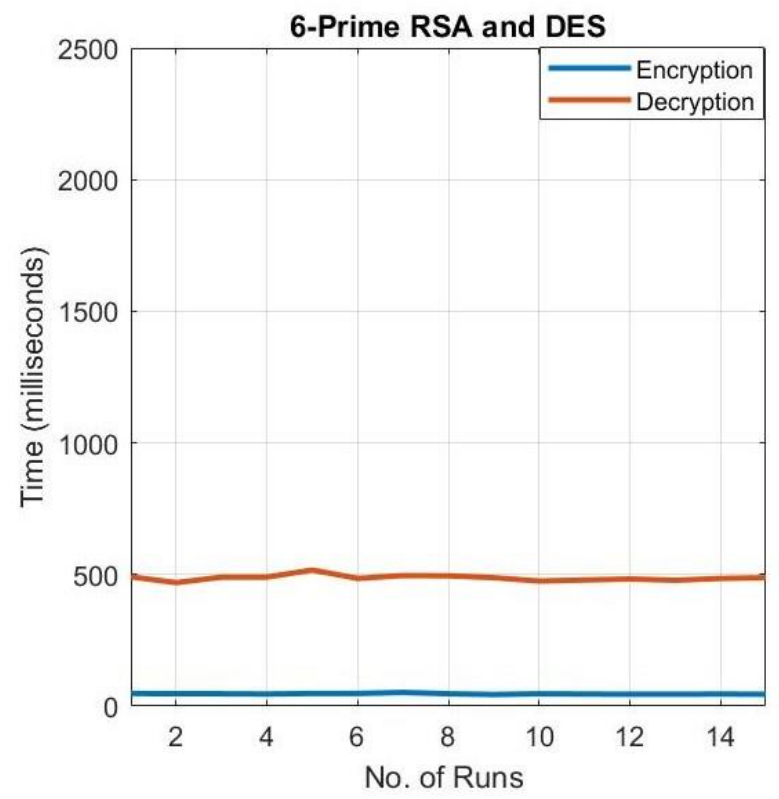

Fig.8. Encryption/Decryption Times for 6-prime RSA+DES.

\section{E. DES and 7-Prime RSA}

The 7-prime RSA encryption is used in place of the Standard RSA. The DES encryption/decryption remains the same.

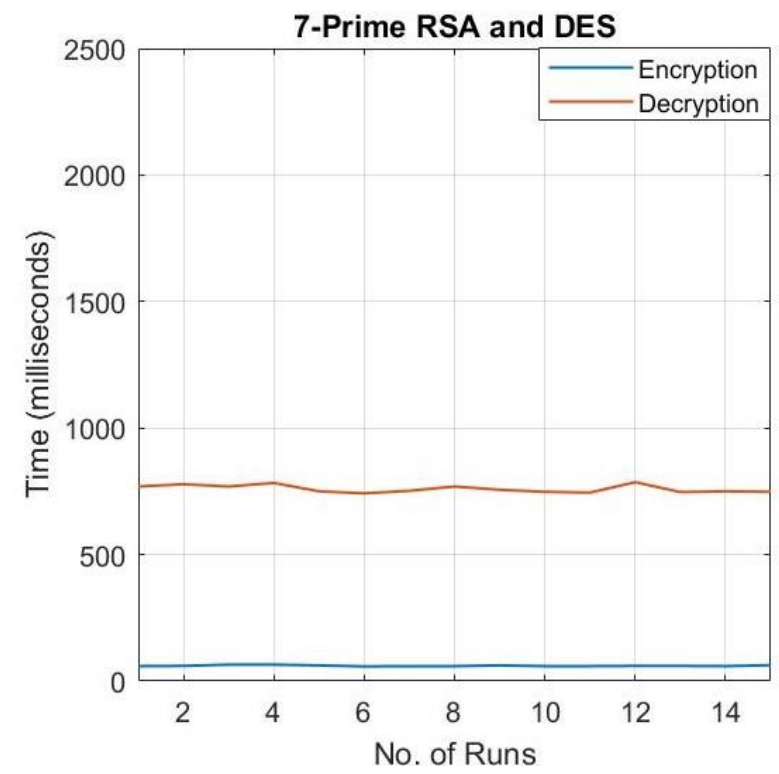

Fig.9. Encryption/Decryption Times for 7-prime RSA+DES.

\section{F. DES and 8-prime RSA}

The 8-prime RSA encryption is used in place of the Standard RSA. The DES encryption/decryption remains the same. 


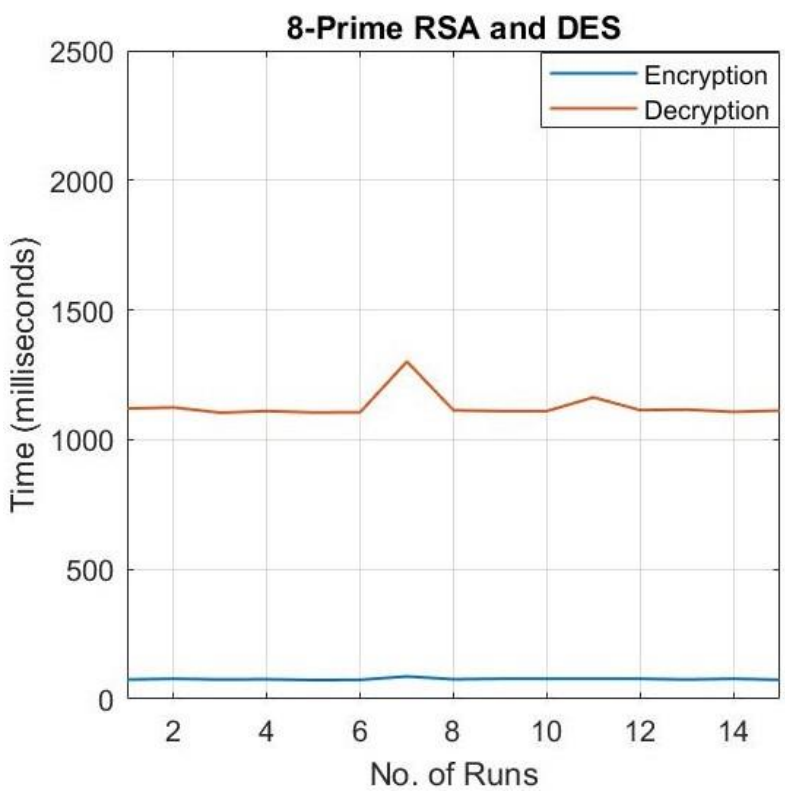

Fig.10. Encryption/Decryption Times for 8-prime RSA+DES.

\section{G. DES and 9-Prime RSA}

The 9-prime RSA encryption is used in place of the Standard RSA. The DES encryption/decryption remains the same.

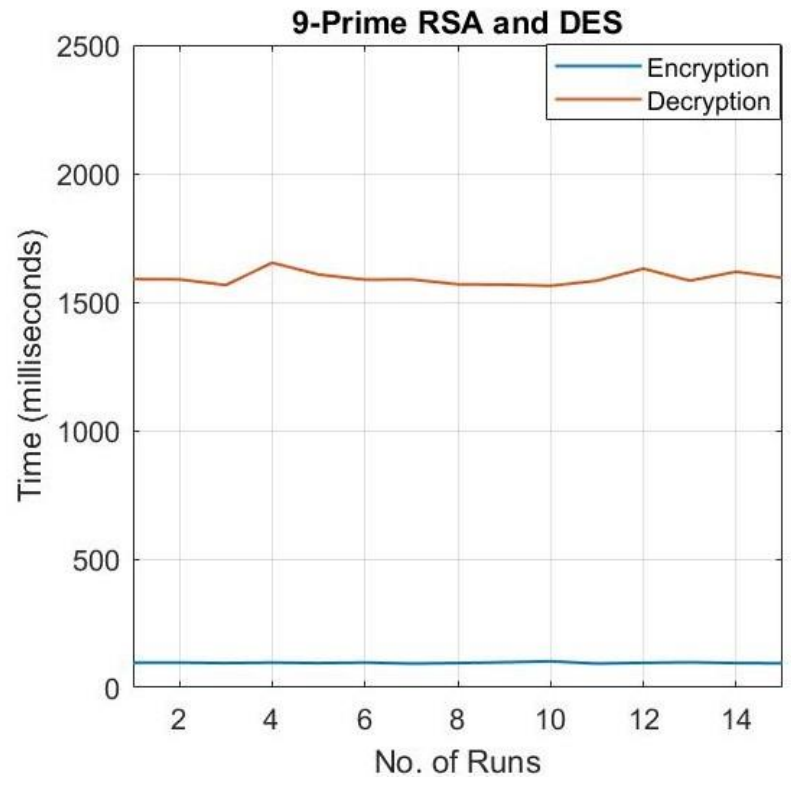

Fig.11. Encryption/Decryption Times for 9-prime RSA+DES

\section{H. DES and 10-Prime RSA}

The 10-prime RSA encryption is used in place of the Standard RSA. The DES encryption/decryption remains the same.

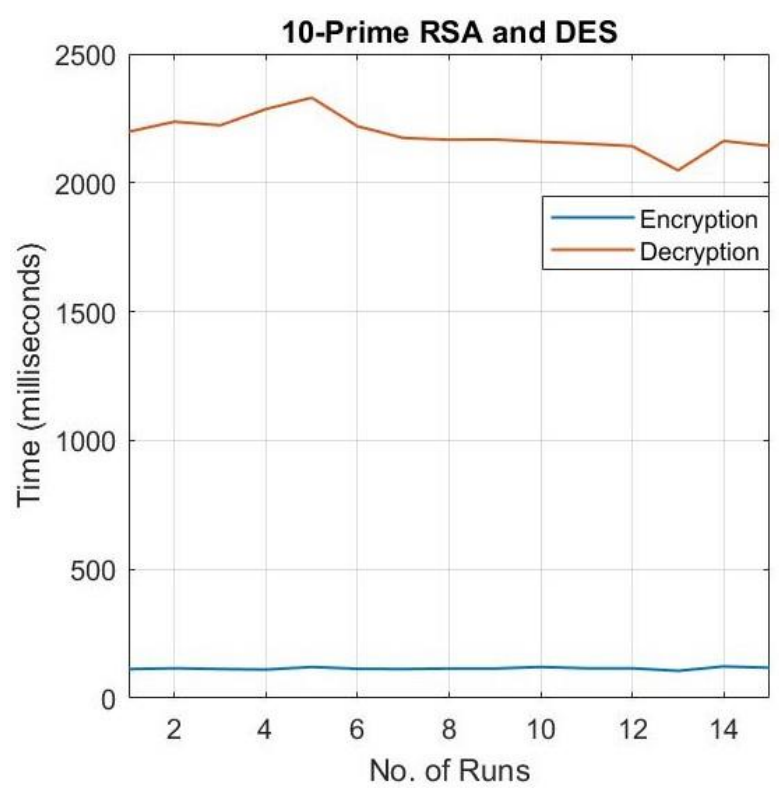

Fig.12. Encryption/Decryption Times for 10-prime RSA+DES.

\section{RESULTS}

Following is the tabular representation of the average encryption and decryption time values for the various versions of RSA algorithm that have been used to encrypt the DES key. The table also shows the average memory consumed by each of these programs.

Table 1. Average Encryption / Decryption Time Values, and Memory Consumption of Combined Multi-Prime RSA and DES.

\begin{tabular}{|l|l|l|l|}
\hline $\begin{array}{l}\text { No. of primes } \\
\text { used in RSA }\end{array}$ & $\begin{array}{l}\text { Average } \\
\text { Encryption } \\
\text { Time (ms) }\end{array}$ & $\begin{array}{l}\text { Average } \\
\text { Decryption } \\
\text { Time (ms) }\end{array}$ & $\begin{array}{l}\text { Average } \\
\text { memory } \\
\text { used. (MB) }\end{array}$ \\
\hline 2 & 14.133333 & 31.333333 & 24.08738556 \\
\hline 3 & 17.266666 & 72.933333 & 27.12103221 \\
\hline 4 & 25.2 & 156.666666 & 7.463562012 \\
\hline 5 & 34.733333 & 290.133333 & 15.43968793 \\
\hline 6 & 46.733333 & 487.266666 & 23.91811218 \\
\hline 7 & 60.6 & 759.466666 & 30.69079132 \\
\hline 8 & 76.866666 & 1127.666666 & 6.035212708 \\
\hline 9 & 96.133333 & 1592.4 & 13.80965068 \\
\hline 10 & 115.4 & 2187.2 & 23.39605459 \\
\hline
\end{tabular}

From the above data, the relation between the no. of primes used in RSA and the observed encryption and decryption times can be illustrated as in fig. 13 .

Clearly, there is increase in both times as the number of prime numbers is increased. However, the increase in decryption time is much more drastic than the encryption time (Fig. 14). From the graph we can infer that combining DES with multi-prime RSA becomes less and less feasible as we increase the number of prime numbers in the RSA section. 


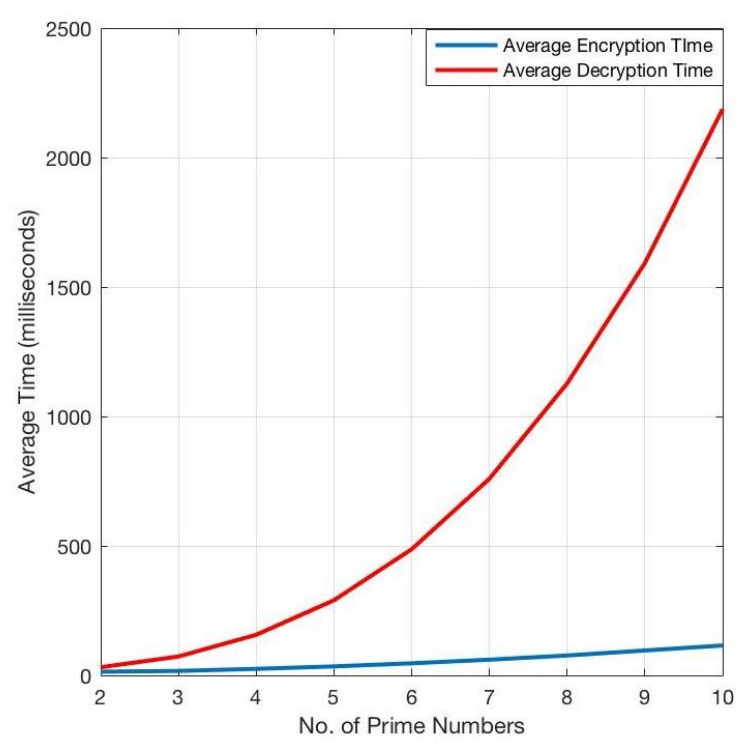

Fig.13. Plot of Avg. Encryption and Decryption time of the RSA+DES Program with Different Number of Primes.

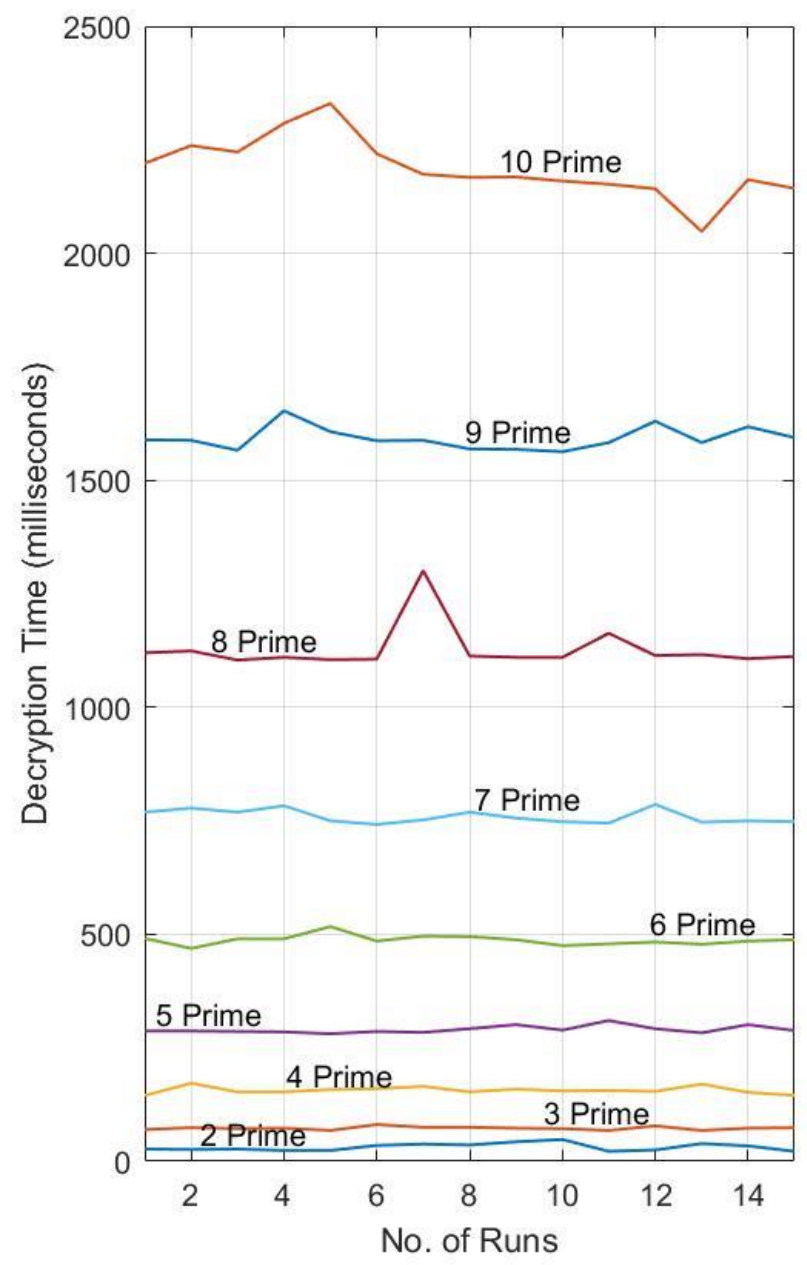

Fig.14. Plot of Decryption times of the RSA+DES Program with Different Number of Primes.

We can also observe the memory consumption with respect to the change in the number of primes in Fig. 15.

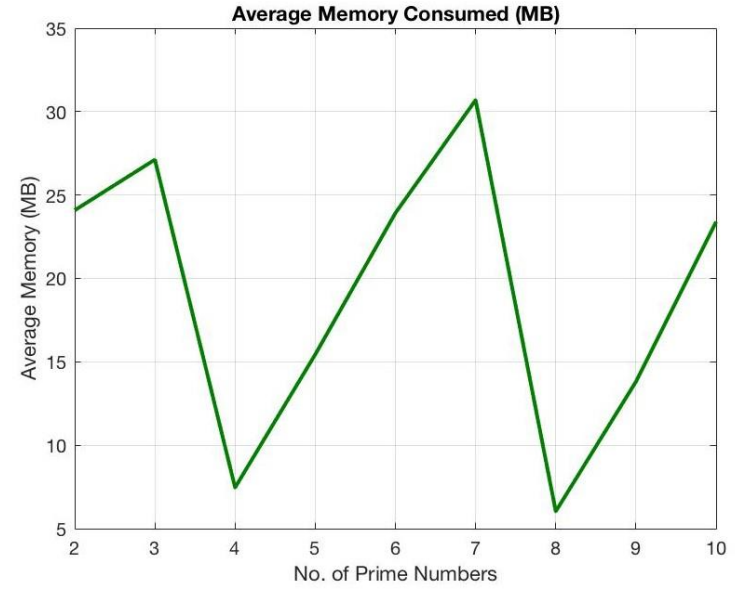

Fig.15. Plot of Avg. Memory consumed by the RSA+DES program w.r.t no. of primes used in the RSA.

What seems like a meaningless trend at first, shows the following vague pattern on closer examination: the consumption for 4 and 8 primes is comparable, for 2,6 and 10 is comparable, for 5 and 9 is also comparable, and 3 and 7 seem separate with no similar consumption figures. (The word 'comparable' has been used here to put together readings with difference less than $1.7 \mathrm{MB}$ )

If we further exclude the standard, i.e. 2 prime RSA from this analysis, we observe that the similarity is occurring at a difference of 4 , in the number of primes used. The exception to this is the 3 and 7 prime consumption values, which seem to have more difference than the rest of the 4-difference pairs. However, we also need to consider that with this vague trend, experimentation with up to 10 prime numbers is not enough to draw actual conclusions.

\section{FUTURE SCOPE}

The rate of change of time with respect to the number of prime numbers in the multilevel algorithm is clearly illustrated in the above graphs. We have experimented with 10 prime numbers and all follow a similar time complexity pattern However, the same cannot be said for the memory usage of the multilevel algorithm.

The memory usage, roughly, alternatively varies with increase of prime numbers, or with a difference of 4 , as explained in the previous section. Due to lack of a clear pattern in memory usage, experimentation with more number of primes is required to get a clear picture of a trend, if any.

\section{LIMITATIONS}

As the prime numbers are increased in the RSA public key encryption, it is observed that the experimental values of encryption time, decryption time, and memory consumption become more and more inconsistent and varying. To illustrate this more clearly, Table 2 presents the experimental values for the 10 prime case. 
Table 2. Encryption, Decryption Time Values, and Memory Consumption of Combined 10-Prime RSA and DES.

\begin{tabular}{|c|c|c|c|}
\hline $\begin{array}{c}\text { Sr. } \\
\text { No. }\end{array}$ & $\begin{array}{c}\text { Encryption } \\
\text { Time }\end{array}$ & $\begin{array}{c}\text { Decryption } \\
\text { Time }\end{array}$ & Memory Consumption \\
\hline 1 & 113 & 2198 & $19.089691162109375 \mathrm{Mb}$ \\
\hline 2 & 116 & 2237 & $22.889625549316406 \mathrm{Mb}$ \\
\hline 3 & 113 & 2223 & $23.53436279296875 \mathrm{Mb}$ \\
\hline 4 & 111 & 2286 & $28.833465576171875 \mathrm{Mb}$ \\
\hline 5 & 121 & 2330 & $27.58641815185547 \mathrm{Mb}$ \\
\hline 6 & 114 & 2219 & $22.204750061035156 \mathrm{Mb}$ \\
\hline 7 & 113 & 2174 & $18.764930725097656 \mathrm{Mb}$ \\
\hline 8 & 115 & 2167 & $20.98853302001953 \mathrm{Mb}$ \\
\hline 9 & 115 & 2168 & $22.851524353027344 \mathrm{Mb}$ \\
\hline 10 & 121 & 2159 & $24.183029174804688 \mathrm{Mb}$ \\
\hline 11 & 116 & 2152 & $20.977310180664062 \mathrm{Mb}$ \\
\hline 12 & 116 & 2142 & $22.871498107910156 \mathrm{Mb}$ \\
\hline 13 & 106 & 2048 & $24.81622314453125 \mathrm{Mb}$ \\
\hline 14 & 123 & 2162 & $26.0682373046875 \mathrm{Mb}$ \\
\hline 15 & 118 & 2143 & $25.281219482421875 \mathrm{Mb}$ \\
\hline
\end{tabular}

Further, actual memory consumption and time values are largely dependent on the processor capabilities of the system used. The data illustrated in this paper has been compiled on the Mac OS, but the values will change if another OS/processor is used. At the same time, it is important to note that the trends of increase and decrease in values would essentially remain the same.

\section{CONCLUSION}

A widely used public key encryption algorithm, RSA, when combined with a symmetric key algorithm, DES, has its perks, but when Multi-Prime RSA is combined with DES, it definitely proves to be advantageous [15]. The decryption time of Multi-Prime RSA, especially 3Prime and 4-prime is much more than the decryption time of standard RSA. The major disadvantage of DES is the limited key size, hence, it can be deciphered through brute-force attacks. The multi-level RSA and DES overcome this disadvantage by encrypting the DES key, therefore, making it more secure and reliable. As shown in graphs in section IV, the decryption time significantly increases as number of primes increase, but as compared to it the encryption time increases at a much slower pace. Also, the memory utilization alternatively increases and decreases as we move from 2 to 10 prime numbers. Under the binate protection of DES and Multi-Prime RSA, the data becomes more secure.

\section{ACKNOWLEDGMENT}

We sincerely thank our guide and mentor, Surinder Kaur, Assistant Professor at BVCOE, for her immense support and guidance, and for constantly giving us sound advice and motivation.

\section{REFERENCES}

[1] H E. Milanov, "The RSA algorithm," June 2009, 2009.

[2] H. M. Bahig, A. Bhery, and D. I. Nassr. Cryptanalysis of multi-prime RSA with small prime difference. In Information and Communications Security, pages 33-44. Springer, 2012.

[3] Alani, M.M.," A DES96 - improved DES security ", 7th International Multi-Conference on Systems, Signals and Devices, Amman, 27-30 June 2010

[4] Chourasia S., Singh K.N. (2016) An Efficient Hybrid Encryption Technique Based on DES and RSA for Textual Data. In: Satapathy S., Mandal J., Udgata S., Bhateja V. (eds) Information Systems Design and Intelligent Applications. Advances in Intelligent Systems and Computing, vol 433. Springer, New Delhi.

[5] Wuling Ren; Zhiqian Miao, "A Hybrid Encryption Algorithm Based on DES and RSA in Bluetooth Communication," Modeling, Simulation and Visualization Methods (WMSVM), Second International Conference on, vol., no., pp. 221, 225, 15-16 May 2010.

[6] Coppersmith, D. "The Data Encryption Standard (DES) and Its Strength Against Attacks." IBM Journal of Research and Development, May 1994, pp. 243 -250.

[7] Atul Kahate, "Cryptography and Network Security, 2nd Ed," Tata McGraw hill, 2009, PP. 87-2004.

[8] Chourasia S., Singh K.N. (2016) An Efficient Hybrid Encryption Technique Based on DES and RSA for Textual Data. In: Satapathy S., Mandal J., Udgata S., Bhateja V. (eds) Information Systems Design and Intelligent Applications. Advances in Intelligent Systems and Computing, vol 433. Springer, New Delhi.

[9] A. A. A. Gutub and F. A. A. Khan, "Hybrid Crypto Hardware Utilizing Symmetric-Key and Public-Key Cryptosystems," 2012 International Conference on Advanced Computer Science Applications and Technologies (ACSAT), Kuala Lumpur, 2012, pp. 116-121.

[10] Kuppuswamy, Prakash, Khalidi, Saeed Q.Y., "Hybrid encryption/decryption technique using new public key and symmetric key algorithm", International Journal of Information and Computer Security pg.372-382, 2014/01/01.

[11] Amanjot Kaur, Manisha Bhardwaj, "Hybrid Encryption for Cloud Database Security", [IJESAT] International Journal of Engineering Science \& Advanced Technology, Volume-2, Issue-3, 737 - 741, ISSN: 2250-3676.

[12] W. Ren and Z. Miao, "A Hybrid Encryption Algorithm Based on DES and RSA in Bluetooth Communication," 2010 Second International Conference on Modeling, Simulation and Visualization Methods, Sanya, 2010, pp. 221-225.

[13] Rivest, R. L., Shamir, A., Adelmann, L.: "A method for obtaining digital signature and public -key cryptosystems", Commun. ACM, 1978, VOL. 21, pp. 120-126.

[14] Penchalaiah, N. and Seshadri, R. "Effective Comparison and Evaluation of DES and Rijndael Algorithm (AES)", International Journal of Computer Science and Engineering, Vol. 02, No. 05, 2010, 1641-1645.

[15] Stallings, William; "Cryptography and Network Security Principles and Practices"; Fourth Edition; Pearson Education; Prentice Hall; 2009. 


\section{Authors' Profiles}

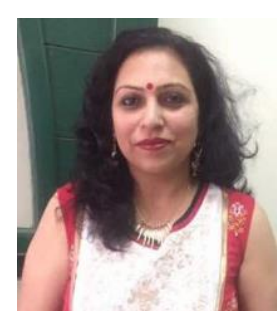

Surinder Kaur is currently an Assistant Professor at Bharati Vidyapeeth's College of Engineering, affiliated to GGSIPU, New Delhi. She currently has three publications including one in INDIACom 2017, an IEEE conference.

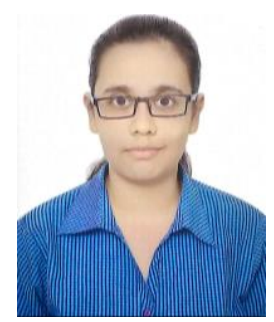

Pooja Bharadwaj is currently pursuing B. Tech in Information Technology from Guru Gobind Singh Indraprastha University, batch of 2013-17. She has one published survey paper on cloud security and another research paper that has been accepted and presented at INDIACom 2017, an IEEE conference.

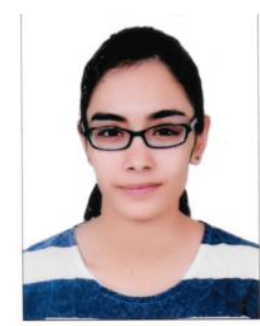

Shivani Mankotia is currently pursuing B. Tech in Information Technology from Guru Gobind Singh Indraprastha University, batch of 2013-17. She has one published survey paper on cloud security and another research paper that has been accepted and presented at INDIACom 2017, an IEEE conference.

How to cite this paper: Surinder Kaur, Pooja Bharadwaj, Shivani Mankotia,"Study of Multi-Level Cryptography Algorithm: Multi-Prime RSA and DES", International Journal of Computer Network and Information Security(IJCNIS), Vol.9, No.9, pp.22-29, 2017.DOI: 10.5815/ijcnis.2017.09.03 